\section{El protocolo de investigación III: la población de estudio}

Jesús Arias-Gómez, ${ }^{1}$ Miguel Ángel Villasís-Keever, ${ }^{2}$ María Guadalupe MirandaNovales ${ }^{3}$

\section{Resumen}

La población de estudio es un conjunto de casos, definido, limitado y accesible, que formará el referente para la elección de la muestra que cumple con una serie de criterios predeterminados. Los objetivos de este artículo están dirigidos a especificar cada uno de los elementos que se requiere tomar en cuenta para la selección de los participantes de una investigación, en el momento en que se está elaborando un protocolo, donde se incluyen los conceptos de población de estudio, muestra, criterios de selección y técnicas de muestreo. Posterior a definir la población de estudio, el investigador debe especificar los criterios a cumplir por los participantes. Los criterios que especifican las características que la población debe tener se denominan criterios de elegibilidad o de selección. Estos criterios son los de inclusión, exclusión y eliminación, que delimitan la población elegible. Los procedimientos de muestreo se dividen en dos grandes grupos: 1) muestreos probabilísticos o aleatorios y 2) muestreo no probabilístico. La diferencia entre ambos está dada por la utilización de métodos estadísticos para la elección de los sujetos. En toda investigación siempre debe determinarse, desde el principio, el número específico de participantes que será necesario incluir a fin de lograr los objetivos planteados. Este número se conoce como tamaño de muestra, que se estima o calcula mediante fórmulas matemáticas o paquetes estadísticos.

PALABRAS CLAVE: población de estudio, criterios de selección, muestreo, tamaño de muestra.

Rev Alerg Méx 2016 Apr-Jun;63(2):201-206.

\section{The research protocol IIII. Study population}

Jesús Arias-Gómez, ${ }^{1}$ Miguel Ángel Villasís-Keever, ${ }^{2}$ María Guadalupe MirandaNovales $^{3}$

\section{Abstract}

The study population is defined as a set of cases, determined, limited, and accessible, that will constitute the subjects for the selection of the sample, and must fulfill several characteristics and distinct criteria. The objectives of this manuscript are focused on specifying each one of the elements required to make the selection of the participants of a research project, during the elaboration of the protocol, including the concepts of study population, sample, selection criteria and sampling methods. After delineating the study population, the researcher must specify the criteria that each participant has to comply. The criteria that include the specific characteristics are denominated selection or eligibility criteria. These criteria are inclusion, exclusion and elimination, and will delineate the eligible population. The sampling methods are
${ }^{1}$ Casa Cuna Tlalpan, Desarrollo Integral de la Familia (DIF), Ciudad de México, México.

2 Unidad de Investigación en Epidemiología Clínica, Hospital de Pediatría, Coordinación de Investigación en Salud, Centro Médico Nacional Siglo XXI, Instituto Mexicano del Seguro Social, Ciudad de México.

${ }^{3}$ Unidad de Investigación en Epidemiología Hospitalaria, Hospital de Pediatría, Coordinación de Investigación en Salud, Centro Médico Nacional Siglo XXI, Instituto Mexicano del Seguro Social, Ciudad de México.

Recibido: 1 de abril 2016

Aceptado: 2 de abril 2016

Correspondencia

Dra. María Guadalupe Miranda-Novales

Teléfono: (55) 5627 6900, extensión 22507

guadalumiranda@terra.com.mx

Este artículo debe citarse como

Arias-Gómez J, Villasís-Keever MÁ, Miranda-Novales MG. El protocolo de investigación III: la población de estudio. Rev Alerg Méx. 2016 abr-jun;63(2):201-206. 
divided in two large groups: 1) probabilistic or random sampling and 2) non-probabilistic sampling. The difference lies in the employment of statistical methods to select the subjects. In every research, it is necessary to establish at the beginning the specific number of participants to be included to achieve the objectives of the study. This number is the sample size, and can be calculated or estimated with mathematical formulas and statistic software.

KEYWORDS: study population, selection criteria, sampling, sample size.

\section{Correspondence}

Dra. María Guadalupe Miranda-Novales Teléfono: (55) 5627 6900, extensión 22507 guadalumiranda@terra.com.mx

\section{INTRODUCCIÓN}

La integración del grupo de sujetos o participantes de los estudios, con las características particulares que permitirán responder los objetivos planteados, es una parte fundamental de todo protocolo de investigación porque cuando se logra una apropiada selección, no solo se podrá disponer de resultados confiables, sino que es posible que dichos resultados puedan ser extrapolados a otras poblaciones similares. Además, una buena elección de los participantes para el proyecto cumple con el propósito esencial de asegurar que los hallazgos representarán, de forma exacta, lo que sucede en la población de interés.

Los objetivos de este artículo están dirigidos a especificar cada uno de los elementos que se requiere tomar en cuenta para la selección de los participantes de una investigación, en el momento en que se está elaborando un protocolo, donde se incluyen los conceptos de población de estudio, muestra, criterios de selección y técnicas de muestreo.

\section{POBLACIÓN DE ESTUDIO}

La población de estudio es un conjunto de casos, definido, limitado y accesible, que formará el referente para la elección de la muestra, y que cumple con una serie de criterios prede- terminados. Es necesario aclarar que cuando se habla de población de estudio, el término no se refiere exclusivamente a seres humanos sino que también puede corresponder a animales, muestras biológicas, expedientes, hospitales, objetos, familias, organizaciones, etc.; para estos últimos, podría ser más adecuado utilizar un término análogo, como universo de estudio.

Es importante especificar la población de estudio porque al concluir la investigación a partir de una muestra de dicha población, será posible generalizar o extrapolar los resultados obtenidos del estudio hacia el resto de la población o universo. Por ejemplo, si se desea evaluar la evolución de las concentraciones séricas de IgE en pacientes con alergia alimentaria menores de 2 años, entonces la población de estudio estará constituida por los pacientes pediátricos con alergia alimentaria, atendidos en cierto hospital o unidad médica.

Es conveniente que la población o universo se identifique desde los objetivos del estudio, y puede ser en términos clínicos, geográficos, sociales, económicos, etc.

\section{SELECCIÓN DE LA POBLACIÓN A ESTUDIAR}

En general, para cualquier estudio de investigación se incluyen muestras o subgrupos de poblaciones $y$, en pocas ocasiones, la población total o universo 
completo. Las razones para estudiar muestras en lugar de las poblaciones son diversas y entre ellas: a) ahorrar tiempo, estudiar un número menor de individuos necesariamente se realiza en menor tiempo; $b$ ) en consecuencia se ahorran recursos; c) estudiar a la totalidad de los miembros con una característica determinada, en muchas ocasiones puede ser una tarea inaccesible o imposible de realizar; d) aumentar la calidad del estudio, al disponer de más recursos, las observaciones y mediciones efectuadas a un número reducido de individuos pueden ser más exactas; e) la selección de la muestra permitirá reducir la heterogeneidad de una población, y f) en un sentido estricto y ético no es necesario estudiar al total de la población cuando con una proporción de sujetos puede conseguir los objetivos del estudio.

En la selección de la población de estudio existen características decisivas que deben considerarse. Una de ellas es la homogeneidad, que se refiere a que todos los miembros de la población tienen las mismas características según las variables que se habrán de estudiar, ya que si no se asegura que la población sea homogénea puede conducir a elaborar conclusiones equivocadas durante el análisis, ya que por la mezcla de subpoblaciones (heterogéneas) no se obtendrá una representación clara de las variables en estudio.

Otra característica es la temporalidad; es decir, el periodo donde se sitúa a la población de interés. Debe establecerse si el estudio se ubica en el presente, o si se trata de una población atendida en el pasado, o de una conjunción de poblaciones de diferentes generaciones. Esta característica es importante porque las condiciones de las poblaciones pueden variar con el tiempo, ya sea por avances en la forma de establecer diagnósticos o tratamiento, o por los cambios en factores ambientales.

La tercera característica es la necesidad que en la población a estudiar se definan los límites espa- ciales, esto significa que se debe especificar si la población es de una comunidad, país, o unidad médica. En esta última, siempre es conveniente señalar si es de primer, segundo o tercer nivel de atención ya que en cada uno de estos niveles, los pacientes atendidos generalmente son diferentes (por su gravedad, tipo de tratamiento, comorbilidades, entre otros).

Con lo anteriormente expuesto es más claro entender que en cualquier investigación no se estudiará al total de la población, y que solo se elegirá a una fracción o muestra de la población definida en los objetivos. A este respecto diversos autores han propuesto un cambio en la nomenclatura de las poblaciones que se emplean para marcar las diferencias entre una población general y una muestra. Así, se refieren varios tipos de universos: el finito, infinito e hipotético; además se consideran diferentes niveles de población: población diana o blanco, accesible y elegible. Mientras que otros autores las denominan población muestra o población participante. A continuación se describen las definiciones:

El universo finito es aquel donde los elementos que lo constituyen pueden ser delimitados y cuantificados. Como ejemplos: 1) pacientes con diagnóstico de dermatitis atópica de un hospital de tercer nivel de atención, y 2) residentes de la especialidad de Alergia en la Ciudad de México. Se identifica el universo infinito cuando los elementos que lo conforman no tienen límite o en términos prácticos, cuando no es posible determinar su magnitud debido al tamaño. Un ejemplo puede ser, pacientes con asma en la Ciudad de México; otro ejemplo es la población de médicos de un determinado país.

Con respecto al universo hipotético se reconoce cuando el tamaño de la población no es posible definirlo en forma precisa porque se trata de eventos o hechos que aún no han ocurrido. Por ejemplo, el número de pacientes con diagnóstico 
de asma de un determinado hospital durante el segundo semestre del 2016. En este estudio el universo estará conformado por todos los se presenten a partir del 1 de julio del 2016, por lo que no se tiene certeza del número exacto y será necesario la estimación del total a estudiar.

La población diana o blanco se conoce a la delimitación del grupo a estudiar, basado en ciertas características clínicas, demográficas, sociales, estilos de vida, etc. De esta manera, en el ejemplo del estudio con niños con alergia alimentaria se puede agregar quienes tengan manifestaciones más graves de la enfermedad. De esta forma, al ser más específica la población a estudiar, entonces será más probable la generalización de los hallazgos de una investigación. Un subgrupo de la población diana es la que corresponde a población accesible, que se determina por consideraciones prácticas en función de las posibilidades o recursos que dispongan los investigadores. Por ejemplo, es posible que solo se puedan estudiar pacientes con asma que acuden a un hospital de tercer nivel.

\section{Criterios de selección}

Posterior a definir la población de estudio, el investigador debe especificar los criterios que deben cumplir los participantes. Los criterios que especifican las características que la población debe tener se denominan criterios de elegibilidad o criterios de selección. Estos criterios son los criterios de inclusión, exclusión y eliminación, que son los que van a delimitar a la población elegible:

1. Criterios de inclusión: son todas las características particulares que debe tener un sujeto u objeto de estudio para que sea parte de la investigación. Estas características, entre otras, pueden ser: la edad, sexo, grado escolar, nivel socioeconómico, tipo específico de enfermedad, estadio de la enfermedad y estado civil. Además, cuando la población son seres humanos es conveniente señalar la aceptación explícita de su participación mediante carta de consentimiento informado $y$, en caso de niños, de carta de asentimiento.

2. Criterios de exclusión: se refiere a las condiciones o características que presentan los participantes y que pueden alterar o modificar los resultados, que en consecuencia los hacen no elegibles para el estudio. Típicamente estos criterios de exclusión se relacionan con la edad, etnicidad, por la presencia de co-morbilidades, gravedad de la enfermedad, presencia de embarazo, o las preferencias de los pacientes. Es importante destacar que estas características no corresponden a lo "contrario" de los criterios de inclusión; por ejemplo, si en el estudio se define que se incluirán mujeres, en los de exclusión no debe señalarse hombres, o bien, si el estudio será de adultos, no es correcta la exclusión de niños.

3. Criterios de eliminación: Este aspecto corresponde con las características que se pueden presentar en el desarrollo de la investigación. Es decir, serán circunstancias que pueden ocurrir después de iniciar la investigación y de haber seleccionado a los participantes. Por ejemplo, en un estudio longitudinal con la vigilancia mensual de los pacientes durante un año, los pacientes que dejaron de acudir por cualquier causa (entre otras, muerte, cambio de domicilio, no deseo de seguir participando en el estudio) en algún momento, no deberán ser considerados al final, por esta razón serán eliminados del estudio. En el caso de estudios transversales, como en una encuesta, el criterio de eliminación sucede cuando los participantes no completan apropiadamente la o las evaluaciones programadas.

\section{Método de selección o técnicas de muestreo}

Una vez definidas las características de los participantes en el estudio, es necesario que se 
garantice, en la medida de lo posible que dicha muestra sea representativa de la población de estudio. Como se comentó, los resultados de toda investigación deberían poder generalizarse en vista que no se puede estudiar al total de la población, es decir, que se puedan hacer inferencias a partir de la muestra estudiada. La mejor forma de hacerlo es que la muestra de participantes sea elegida de manera aleatoria, con el propósito que todos los elementos de la población tengan la misma probabilidad de ser incluidos en el estudio. Sin embargo, esto no es posible realizarlo en todos los estudios por diferentes razones, por lo cual se necesario recurrir a los procedimientos denominados técnicas de muestreo; según la técnica de muestreo empleada podremos tener mayor o menor seguridad en cuanto a que la muestra sea representativa.

Los procedimientos de muestreo se dividen en dos grandes grupos: 1) los muestreos probabilísticos o aleatorios y 2) muestreo no probabilístico. La diferencia entre ambos está dada por la utilización de métodos estadísticos para la elección de los sujetos.

Dentro de los métodos de muestreo probabilísticos encontramos los siguientes tipos:

1. Muestreo aleatorio simple: El procedimiento empleado es el siguiente: 1) se asigna un número a cada individuo de la población y 2) a través de algún medio (tablas de números aleatorios, números aleatorios generados con un programa de computadora, etc.) se eligen tantos sujetos como sea necesario para completar el tamaño de muestra requerido. Este procedimiento, atractivo por su simpleza, tiene poca o nula utilidad práctica cuando la población que estamos manejando es muy grande.

2. Muestreo aleatorio estratificado: trata de obviar las dificultades que presenta el anterior, ya que simplifican los procesos y suelen reducir el error muestral. Consiste en considerar categorías típicas diferentes entre sí (estratos) que poseen gran homogeneidad respecto a alguna característica (se puede estratificar, según la profesión, municipio de residencia, sexo, estado civil, etc.). Lo que se pretende con este tipo de muestreo es asegurarse de que todos los estratos de interés estarán representados adecuadamente en la muestra. Una de las dificultades que se plantea con este tipo de muestreo es la necesidad de disponer de un conocimiento detallado de la población.

3. Muestreo aleatorio por conglomerados: En el muestreo por conglomerados la unidad muestral es un grupo de elementos de la población que forman una unidad, a la que Ilamamos conglomerado. Las unidades hospitalarias, los departamentos universitarios, una caja de determinado producto, etc., son conglomerados naturales. En otras ocasiones se pueden utilizar conglomerados no naturales como, por ejemplo, las urnas electorales. Cuando los conglomerados son áreas geográficas suele hablarse de "muestreo por áreas". El muestreo por conglomerados consiste en seleccionar aleatoriamente un cierto número de conglomerados, a fin de investigar todos los elementos pertenecientes a los conglomerados elegidos.

\section{Métodos de muestreo no probabilísticos}

A veces, para estudios exploratorios, el muestreo probabilístico resulta excesivamente costoso y se acude a métodos no probabilísticos, aun siendo conscientes que no sirven para realizar generalizaciones, pues no se tiene certeza de que la muestra extraída sea representativa. En general se seleccionan a los sujetos siguiendo determinados criterios. A continuación se describen algunos de los métodos de muestreo no probabilísticos más utilizados:

1. Muestreo por cuotas: También denominado en ocasiones "accidental". Se asienta 
generalmente sobre la base de un buen conocimiento de los estratos de la población y/o de los individuos más "representativos" o "adecuados" para los fines de la investigación. Mantiene, por tanto, semejanzas con el muestreo aleatorio estratificado, pero no tiene el carácter de aleatoriedad. En este tipo de muestreo se fijan unas "cuotas" que consisten en un número de individuos que reúnen determinadas condiciones, por ejemplo: 20 niños de 2 a 10 años, de sexo femenino, y residentes en Aguascalientes. Una vez determinada la cuota se eligen los primeros que se encuentren que cumplan esas características.

2. Muestreo intencional o de conveniencia: Consiste en la selección por métodos no aleatorios de una muestra cuyas características sean similares a las de la población objetivo. También puede ser que el investigador seleccione directa e intencionadamente los individuos de la población. El caso más frecuente de este procedimiento es utilizar como muestra los individuos a los que se tiene fácil acceso (por ejemplo, los profesores de universidad emplean con mucha frecuencia a sus propios alumnos, o bien, el número de pacientes que acudió en un tiempo determinado). En general, el método puede resultar de utilidad cuando se pretende realizar una exploración de un fenómeno en una población o cuando no existe un tamaño muestral definido.

3. Bola de nieve: Se localiza a algunos individuos, los cuales conducen a otros, y estos a otros, y así hasta conseguir una muestra suficiente. Este tipo se emplea muy frecuentemente cuando se hacen estudios con poblaciones "marginales", delincuentes, sectas, determinados tipos de enfermos, etc.

\section{Tamaño de muestra}

En toda investigación siempre debe determinarse el número específico de participantes que será necesario incluir a fin de lograr los objetivos planteados desde un principio. Este número se conoce como tamaño de muestra, que se estima o calcula mediante fórmulas matemáticas o paquetes estadísticos. Este cálculo es diferente para cada investigación y depende, entre otras cosas, de su diseño, hipótesis planteadas, número de grupos a estudiar, y de la escala de medición de las variables. En virtud de que es un tema muy amplio y que sale de los objetivos de este artículo, se abordará en el futuro en un artículo subsiguiente. En tanto el lector puede consultar otras fuentes bibliográficas.

\section{BIBLIOGRAFÍA RECOMENDADA}

1. Álvarez Cáceres R. Estadística aplicada a las ciencias de la salud. 1a ed. Madrid: Ediciones Díaz de Santos, 2007

2. Borda Pérez M. Métodos cuantitativos: herramientas para la investigación en salud. 2a ed. Barranquilla, Colombia 2009.

3. Donaldson RJ, Donaldson LJ. Medicina comunitaria. 1a ed. Madrid. Ediciones Díaz Santos, 1989.

4. Gordillo Moscoso AA. Manual de Investigación clínica. 1a ed. México: El Manual Moderno, 2012.

5. Hulley SB, Cummings SR, Browner WS, Grady D, Newman TB. Designing clinical research. 4 th ed. Philadelphia, USA. Lippincott Williams \& Wilkins, 2013.

6. Kothari CR. Research methodology. Methods \& techniques. $2^{\text {nd }}$ ed. New Dehli: New Age International (P) Ltd., Publishers, 2004.

7. Martirosyan L, Arah OA, Haaijer-Ruskamp FM, Braspenning J, Denig P. Methods to identify the target population: implications for prescribing quality indicators. BMC Health Services Research. 2010;10: 137.

8. Talavera JO, Rivas-Ruiz R, Bernal-Rosales LP. Investigación clínica V. Tamaño de muestra. Rev Med Inst Mex Seguro Soc. 2011;49:517-522.

9. Weng Ch, Tu SW, Sim I, Richesson R. Formal representations of eligibility criteria: a literature review. J Biomed Inform 2010; 43: 451-467. 